\title{
Microbiota Isolada de Queijos Artesanais em Quatro Capitais da Região Nordeste do Brasil
}

\author{
Elisa Hizuru Uemura Yamanaka (I,li,lii,Iv), Laura Lúcia Cogo (I), \\ Patricia Dalzoto (I), Fernanda Castelhano De Souza (I), Alessandra \\ Vale Daur Guerim (Iv), Fabiana Nicol Barbieri (Iv), Otacilio Moreira- \\ Filho (Iv), Maire Wakamori (Iv), Carla Fran Silva (I)
}

(I) UFPR - Universidade Federal do Paraná (Av. Cel. Francisco Heráclito dos Santos, 100 - Curitiba, PR), (II) UNIANDRADE - Centro Universitário Campos de Andrade (R. João Scuissiato n¹ - Santa Quitéria - Curitiba PR ), (III) SENAI/PR - SENAI CIC - Serviço Nacional da Indústria (R. Senador Accioly Filho, 298 - Curitiba, PR), 4 LABORCLIN - Laborclin - Produtos para Laboratórios Ltda (R. Casimiro de Abreu, 521 - Pinhais, PR)

\section{Resumo}

Os queijos com alto teor de umidade geralmente representam fonte de renda adicional nas diversas regiões brasileiras devido à facilidade de fabricação ao nível artesanal. A maioria das doenças transmitidas por alimentos no Brasil são causadas por Salmonella spp., Escherichia coli patogênica, e por toxinas de Staphylococcus coagulase positiva. O objetivo do trabalho foi isolar a microbiota a partir de queijos com alto teor de umidade, produzidos artesanalmente, nas áreas metropolitanas de quatro capitais da região nordeste do Brasil. A coleta nestas cidades visou avaliar as condições sanitárias na fabricação de alimentos artesanais, devido ao grande fluxo de turistas nestes locais. Entre fevereiro e maio de 2013 foram coletados 12 queijos produzidos artesanalmente na região metropolitana de Salvador, Recife, Natal e Fortaleza, sedes dos jogos da Copa do Mundo de futebol em 2014. Foram realizadas análises para contagem dos indicadores de contaminação E. coli e Staphylococcus coagulase positiva, e pesquisa de patógenos Salmonella spp. e Listeria monocytogenes, utilizando-se metodologias ISO 16649-2, 6888-1, 6579 e 11290, respectivamente. Considerando E. coli como a principal representante de coliformes a $45^{\circ} \mathrm{C}$, 58\% das amostras apresentaram valores acima dos permitidos pela legislação vigente. Foram isolados Staphylococcus coagulase positiva a

\footnotetext{
Referência:

Elisa Hizuru Uemura Yamanaka, Laura Lúcia Cogo, Patricia Dalzoto, Fernanda Castelhano De Souza, Alessandra Vale Daur Guerim (Iv), Fabiana Nicol Barbieri, Otacilio Moreira-Filho, Maire Wakamori, Carla Fran Silva. Microbiota Isolada de Queijos Artesanais em Quatro Capitais da Região Nordeste do Brasil. In: Anais do 12 Congresso Latinoamericano de Microbiologia e Higiene de Alimentos - MICROAL 2014 [= Blucher Food Science Proceedings, num.1, vol.1]. São Paulo: Editora Blucher, 2014. DOI 10.5151/foodsci-microal-212
} 
partir de 50\% das amostras acima da tolerância permitida em legislação vigente. Dentre os patógenos, apesar de não ter sido isolado L. monocytogenes, foi isolada Salmonella spp. $/ 25 \mathrm{~g}$ de uma amostra. Os dados corroboram com os obtidos por outros autores. Conclui-se que o consumo de queijos artesanais representa risco potencial à saúde do consumidor e ressalta-se a importância de uma fiscalização rigorosa e efetiva. Além disso, deve-se adotar medidas higiênico-sanitárias adequadas, com a implantação de Boas Práticas de Fabricação, em locais de fabricação destes, para evitar a disseminação de doenças através dos alimentos pelos turistas que visitam as diversas regiões do Brasil.

Palavras-Chave: segurança alimentar, Salmonella spp., Listeria monocytogenes, Escherichia coli, Staphylococcus coagualse positiva Agência de Fomento: 